\title{
Vibration Reduction and Performance Enhancement of Helicopter Rotors Using an Active/Passive Approach
}

\author{
Bryan Glaz* Peretz P. Friedmann ${ }^{\dagger}$ and Li Liu \\ Department of Aerospace Engineering, The University of Michigan, Ann Arbor, MI, 48109, USA
}

\begin{abstract}
Active/passive optimization of helicopter rotor blades for vibration reduction and performance enhancement at high advance ratios is studied. Dynamic stall is the dominant source of high vibration levels for this flight regime. In the active/passive approach, active control of vibration and required rotor power is implemented with partial span trailingedge flaps operating according to a closed-loop control algorithm. The blade/flap combination is optimized simultaneously using the Efficient Global Optimization (EGO) algorithm. However, due to strong trade-offs between the objective functions, there is no single best active/passive configuration for reduced vibration levels and power consumption. Therefore, a surrogate based multi-objective function optimization approach is employed to find the active/passive configurations corresponding to the best trade-offs between vibration and performance characteristics.
\end{abstract}

\section{Nomenclature}

\begin{tabular}{|c|c|}
\hline$c$ & Blade chord \\
\hline$C_{W}$ & Helicopter weight coefficient \\
\hline$C_{d 0}$ & Blade profile drag coefficient \\
\hline$C_{d f}$ & Flat plate drag coefficient \\
\hline D & Vector of design variables \\
\hline $\mathrm{E}$ & Young's modulus \\
\hline $\begin{array}{c}F_{4 X}, F_{4 Y} \\
F_{4 Z}\end{array}$ & $4 /$ rev hub shears, non-dimensionalized by $m_{0} \Omega^{2} R^{2}$ \\
\hline$g(\mathbf{D})$ & Constraints \\
\hline $\mathrm{h}$ & Height of the blade cross-section \\
\hline$I_{P}$ & Mass polar moment of inertia of the rotor \\
\hline$J_{\mathrm{ACF}}$ & Active control objective function \\
\hline$J_{P}, J_{V}$ & Rotor power and vibration passive optimization objective functions \\
\hline$m_{0}$ & Baseline mass per unit length \\
\hline $\begin{array}{l}m_{n s} \\
M_{4 X}, M_{4 Y}\end{array}$ & Non-structural mass located at the elastic axis \\
\hline$M_{4 Z}$ & $4 /$ rev hub moments, non-dimensionalized by $m_{0} \Omega^{2} R^{3}$ \\
\hline$N_{b}$ & Number of rotor blades \\
\hline$N_{c}$ & Number of behavior constraints \\
\hline$N_{d v}$ & Number of design variables \\
\hline Q & Weighting matrix on objectives to be reduced \\
\hline$R$ & Blade radius \\
\hline$s$ & Predicted error in surrogate objective function \\
\hline$t_{1}, t_{2}, t_{3}$ & Thicknesses of the blade cross-section, see Fig. 2 \\
\hline$w$ & Weight used in weighted expected improvement function \\
\hline$W$ & Weight on the passive objective functions \\
\hline
\end{tabular}




\begin{tabular}{|c|c|}
\hline$W E I F$ & Weighted expected improvement function \\
\hline $\mathbf{W}_{\mathrm{u}}$ & Weighting matrix on control input \\
\hline $\mathbf{W}_{\alpha}$ & Active control weight setting \\
\hline$x_{1}, x_{2}$ & Cross-sectional dimensions, see Fig. 2 \\
\hline$X_{F A}, Z_{F A}$ & $\begin{array}{l}\text { Longitudinal and vertical offsets between rotor hub and helicopter aer } \\
\text { Fig. } 4\end{array}$ \\
\hline$X_{F C}, Z_{F C}$ & $\begin{array}{l}\text { Longitudinal and vertical offsets between rotor hub and helicopter } \\
\text { Fig. } 4\end{array}$ \\
\hline \multicolumn{2}{|l|}{ Symbols } \\
\hline$\alpha_{d}$ & Flight descent angle, see Fig. 4 \\
\hline$\beta_{p}$ & Blade precone angle \\
\hline$\delta_{f}$ & Flap deflection angle \\
\hline$\lambda_{k}$ & Hover stability eigenvalue for $k^{t h}$ mode \\
\hline$\zeta_{k}, \omega_{k}$ & Real and imaginary parts of $\lambda_{k}$ respectively \\
\hline$\mu$ & Advance ratio \\
\hline$\nu$ & Poisson's ratio \\
\hline$\psi$ & Azimuth angle \\
\hline$\Omega$ & Rotor angular speed \\
\hline$\omega_{F 1}, \omega_{L 1}, \omega_{T 1}$ & Fundamental rotating flap, lead-lag and torsional frequencies, /rev \\
\hline$\omega_{L}, \omega_{U}$ & Lower and upper bounds for frequency constraints, /rev \\
\hline$\rho_{\text {filler }}$ & Material density for non-structural filler mass \\
\hline$\rho_{\text {struct }}$ & Material density for the structural member of the blade \\
\hline$\sigma$ & Rotor solidity \\
\hline$\sigma_{\text {allowable }}$ & Allowable blade stress \\
\hline$\sigma_{Y}$ & Yield stress \\
\hline$\sigma_{x x}, \sigma_{x \eta}, \sigma_{x \zeta}$ & Blade stresses \\
\hline & Built-in pretwist angle at the root \\
\hline$\theta_{1}, \theta_{2}$ & Pretwist angle design variables at the $50 \%$ and $100 \%$ blade stations \\
\hline
\end{tabular}

\section{Introduction}

$\mathrm{V}$ IBRATION is one of the most critical concerns in the design of modern rotorcraft. Stricter demands for customer acceptance require low vibration designs, with the ultimate goal being a "jet smooth" ride. In helicopters, the dominant source of vibration is the rotor, which transfers vibratory loads to the rotor hub and fuselage at harmonics that are predominantly $N_{b} /$ rev, where $N_{b}$ is the number of blades. However, significant increases in operational costs due to vibration reduction approaches would prevent implementation of reduced vibration designs in production helicopters. Therefore, it is imperative that vibration reduction approaches do not increase the power consumption associated with the main rotor.

During the last 25 years, two approaches to rotor vibration reduction have emerged. The first approach is passive and includes structural/multidisciplinary optimization for reducing vibrations. ${ }^{1-3}$ The second approach utilizes active control methods. ${ }^{4}$ The passive approach is used by blade designers to improve the vibration characteristics of the rotor. On the other hand, the active approach using on-blade control has not been implemented on a production helicopter, despite a considerable number of wind tunnel and flight tests which have established the effectiveness of this concept.

In the passive approach based on structural optimization, the vibration reduction problem is formulated as a mathematical optimization problem subject to appropriate constraints. The objective function consists of a suitable combination of the $N_{b} /$ rev hub shears and moments that are computed from an aeroelastic response code; constraints are specified on blade stability margins, frequency, blade geometry, autorotational properties, and blade stresses. The design variables can be dimensions of the blade cross-section, mass and stiffness distributions along the span, or geometrical parameters. Typical levels of vibration reduction have been in the range of $30-60 \%$ relative to baseline levels.

Various studies have also demonstrated that modification of the blade's geometric, mass, and stiffness properties is an effective passive approach to power reduction for hover and forward flight. ${ }^{5-9}$ Typical levels of power reduction using a passive approach have been on the order of $4-6 \%$. However, it should be noted that there was no attempt to identify the designs corresponding to the best trade-offs between vibration and 
performance characteristics at a given flight condition in previous studies.

The aeroelastic response simulations needed for vibratory load and power calculations are computationally expensive due to the complex rotary-wing aerodynamic environment, which means that numerous evaluations of the vibration and performance objective functions are costly. Therefore, the direct combination of the objective functions generated by an aeroelastic response simulation with traditional optimization algorithms is computationally impractical. Moreover, traditional optimization search algorithms can converge to local optima, which are known to occur for this class of problems.

Because of the computational cost associated with rotor blade optimization, a global search of the design space requires the use of computationally efficient approximations, or surrogates, in which the objective function is approximated over the entire design space. ${ }^{10}$ The effectiveness of surrogate-based optimization (SBO) for locating reduced vibration designs at low advance ratios in which blade-vortex interaction (BVI) is the dominant source of vibrations, and at high advance ratios where dynamic stall induces significant vibration levels, has been demonstrated in recent studies. ${ }^{11-13}$ References 11 - 13 were based on a comprehensive helicopter simulation code, which is capable of modeling free wake and dynamic stall. In Ref. 13, the Efficient Global Optimization (EGO) algorithm was utilized because it facilitates a global search of the design space with a relatively small number of expensive objective function evaluations, while avoiding poor designs due to inaccuracies in the approximate objective function. The superiority of EGO compared to conventional surrogate based optimization which does not account for the uncertainty in the surrogate's predictions was demonstrated in Ref. 13.

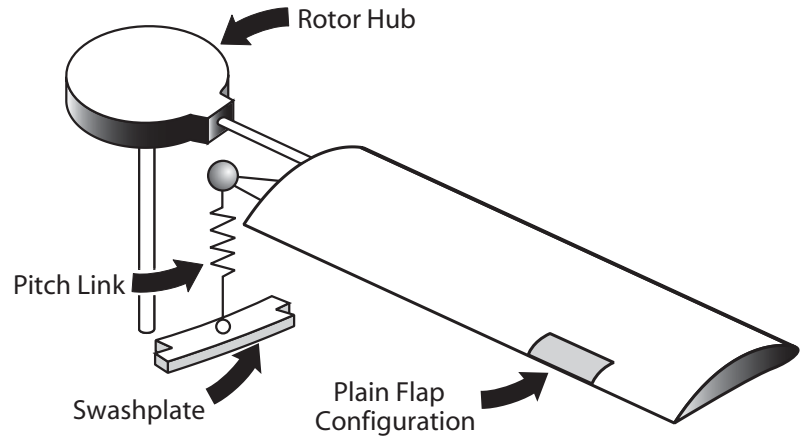

Figure 1. Helicopter rotor blade with partial span trailing edge flap.
Active control approaches to vibration reduction have also been developed. ${ }^{4}$ Over $80 \%$ vibration reduction has been demonstrated by these approaches. Actively controlled flaps (ACF's), as depicted in Fig. 1, have emerged as an attractive means of active control due to their lower power requirement compared to blade root actuation approaches, ${ }^{4}$ and their effectiveness for reducing vibration due to blade-vortex interaction (BVI) and dynamic stall. ${ }^{14,15}$ However, use of ACF's implies a potential performance penalty due to the drag increase associated with flap deflection. Reduction of this penalty is critical for practical implementation of the ACF system in production helicopters. In

Ref. 16, simultaneous vibration reduction and performance enhancement was examined when using ACF's. It was found that vibration reduction of about $50 \%$ could be achieved in conjunction with significant rotor power reduction at a cruising flight speed in which rotor performance is critical.

A judicious combination of active and passive approaches is needed for optimal rotor design. The effectiveness of an active/passive approach to vibration reduction based on structural optimization and ACF's has been demonstrated in previous studies. ${ }^{17-19}$ In Refs. 17 and 18, the optimization algorithm was based on the method of feasible directions, ${ }^{20}$ which is a local search method. The results showed that the active/passive method can result in much lower vibration levels compared to utilization of only one approach. Furthermore, it was demonstrated that optimizing the blade/flap combination simultaneously can lead to superior levels of vibration reduction compared to the sequential approach of adding ACF's to structurally optimized designs. However, the simultaneous optimization approach employed in Ref. 17 was not formulated for multi-objective function design applications. In Ref. 19, a multi-objective function optimization approach based on EGO was developed to identify the best active/passive configurations for reducing BVI induced vibration and noise. Such an approach has yet to be utilized for vibration and power reduction.

The objectives of this paper are:

1. Demonstrate the effectiveness of EGO for structural optimization of the rotor blade to minimize rotor power consumption at high advance ratios in which dynamic stall effects are significant.

2. Examine optimal designs for vibration and power reduction and determine whether these impose conflicting design requirements.

3. Employ a surrogate based multi-objective optimization approach to identify the designs corresponding to the best trade-offs between vibration and performance characteristics. 
4. Demonstrate the effectiveness of augmenting structurally optimized designs with ACF's and controlling for power and vibration reduction.

5. Examine the benefits of a combined active/passive optimization approach in which the blade/flap combination is optimized simultaneously for the best trade-offs between vibration and power reduction.

\section{Description of the Simulation Code Components}

The simulation code used in this study is based on a comprehensive aeroelastic analysis code. ${ }^{21-23}$ The aeroelastic response analysis can represent the behavior of hingeless, bearingless, or articulated rotor blades with actively controlled flaps. The key ingredients of the simulation code are: (1) the structural dynamic model, (2) the unsteady aerodynamic model, and (3) a coupled trim/aeroelastic response solution required for the computation of the blade response. The aeroelastic response analysis, blade stress calculations, and aeroelastic stability in hover analysis are concisely described next.

\section{II.A. Structural Dynamic Model}

The structural dynamic model is based on an analysis developed by Yuan and Friedmann ${ }^{21,24}$ which is capable of modeling composite blades with transverse shear deformations, cross-sectional warping, and swept tips. This study is limited to the behavior of isotropic blades with spanwise varying properties. The equations of motion are formulated using a finite element discretization of Hamilton's principle, with the assumption that the blade undergoes moderate deflections. The beam type finite elements used for the discretization have 23 nodal degrees of freedom. Normal modes are used to reduce the number of structural degrees of freedom. In this study, eight modes are used: the first 3 flap modes, first 2 lead-lag modes, first 2 torsional modes, and the first axial mode. It is assumed that the flap does not change the structural properties of the blade. Thus, only the inertial and aerodynamic effects associated with the presence of the flaps are incorporated in the aeroelastic model.

\section{II.B. Aerodynamic Model}

The attached flow blade section aerodynamics are calculated using a rational function approach (RFA). ${ }^{22}$ The RFA approach is a two-dimensional unsteady time-domain theory that accounts for compressibility as well as variations in the oncoming flow velocity. This two-dimensional aerodynamic model is linked to an enhanced free-wake model which provides a non-uniform inflow distribution at closely spaced azimuthal steps. ${ }^{14,25,26}$ For the separated flow regime, unsteady aerodynamic loads are calculated using the ONERA dynamic stall model described in Ref. 27. The aerodynamic states associated with RFA attached flow and ONERA separated flow are combined to produce the time-domain, state space aerodynamic model. Furthermore, a simple linear drag model which accounts for increase in drag due to flap deflection is implemented. ${ }^{15}$

\section{II.C. Coupled Trim/Aeroelastic Response}

The final set of equations required for the simulation of the aeroelastic response is represented by a system of coupled ordinary differential equations which are cast into first order state variable form and integrated in the time domain using the Adams-Bashforth predictor-corrector algorithm. ${ }^{28}$ A propulsive trim procedure $^{29}$ is used where six equilibrium equations (three forces and three moments) for the entire helicopter in a level steady flight condition are enforced. The same trim conditions as the baseline design are maintained throughout the passive and active control studies. The trim equations are fully coupled with the aeroelastic equations of motion during the response solution. The vibratory hub shears and moments are obtained from the integration of the distributed inertial and aerodynamic loads over the entire blade span in the rotating frame. Subsequently, the loads are transformed to the hub-fixed non-rotating system, and the contributions from the individual blades are combined. ${ }^{24}$ In this process, the blades are assumed to be identical. Cancellation of various terms occurs and the dominant components of the hub shears and moments have a frequency of $N_{b} / \mathrm{rev}$, which is the blade passage frequency. 


\section{II.D. Blade Stresses}

After the blade responses are obtained from the coupled trim/aeroelastic response solution, the stresses in the blade at any spanwise location can be recovered by using strain-displacement and constitutive relations. Solving for the stresses in this manner accounts for the complicated loading a blade encounters and is consistent with the structural dynamic model. The procedure for calculating stresses is as follows:

1. For a given azimuth angle, the displacements at any spanwise location are calculated by the aeroelastic response code.

2. The displacements are then substituted into the nonlinear strain-displacement relations, ${ }^{24}$ giving the strains at any spanwise location.

3. Stresses are calculated from the stress-strain relations.

This calculation gives the blade stresses at any spanwise location and at any azimuth angle.

\section{II.E. Aeroelastic Stability in Hover}

Previous research on optimization of soft-in-plane blades for vibration reduction in forward flight, ${ }^{24}$ has indicated that the aeroelastic stability constraint can be adequately represented by a hover aeroelastic stability constraint, provided that the aeroelastic stability in forward flight is checked for the final blade design. Thus, the method used in this paper is described below:

1. The non-linear static equilibrium solution of the blade is found for a given pitch setting and uniform inflow, by solving a set of nonlinear algebraic equations. Note that uniform inflow is used only in the hover stability calculation. The forward flight analysis employs a free-wake model for inflow calculation.

2. The governing system of ordinary differential equations are linearized about the static equilibrium solution by writing perturbation equations and neglecting second-order and higher terms in the perturbed quantities. The linearized equations are rewritten in first-order state variable form.

3. The real parts of the eigenvalues of the first-order state variable matrix, $\lambda_{k}=\zeta_{k}+i \omega_{k}$, determine the stability of the system. If $\zeta_{k} \leq 0$ for all $k$, the system is stable.

For this study, the linearization process in Ref. 24 is modified to account for the aerodynamic states introduced by the RFA model. ${ }^{11}$

\section{Active/Passive Optimization}

Two approaches to active/passive optimization are employed in this study: the sequential approach and the combined approach. In the sequential approach, structurally optimized blades are augmented with trailing edge flaps. While the passively optimized designs correspond to the best vibration and performance characteristics without the benefits of active control, they do not necessarily result in the greatest levels of improvement when combined with active control. This was demonstrated in Ref. 17 for the case of vibration reduction. Therefore, as an alternative to sequential active/passive optimization, a combined approach is considered in which the blade/flap combination is optimized simultaneously. The resulting designs from the combined approach correspond to the maximum levels of vibration and power reduction when utilizing active control. Descriptions of the passive optimization problem formulation, the passive reduction approach based on EGO, the control algorithm used for active reduction, as well as the sequential and combined active/passive approaches are given next.

\section{III.A. Passive Optimization Problem Formulation}

The formulation of the blade optimization problem in forward flight consists of several ingredients: the objective function, design variables, and constraints. The mathematical formulation of the optimization is stated as: Find the vector of design variables $\mathbf{D}$ which minimizes the objective function, i.e. $J(\mathbf{D}) \rightarrow$ min.

The vibration objective function consists of a combination of the $N_{b} /$ rev oscillatory hub shears and moments. For a four bladed rotor, the vibration objective function is given by

$$
J_{V}=K_{S} \sqrt{\left(F_{4 X}\right)^{2}+\left(F_{4 Y}\right)^{2}+\left(F_{4 Z}\right)^{2}}+K_{M} \sqrt{\left(M_{4 X}\right)^{2}+\left(M_{4 Y}\right)^{2}+\left(M_{4 Z}\right)^{2}}
$$


where $K_{S}$ and $K_{M}$ are appropriately selected weighting factors.

The power objective function used in this study is given by

$$
J_{P}=\frac{\Omega}{2 \pi} \int_{0}^{2 \pi}-M_{H_{Z}}(\psi) \mathrm{d} \psi,
$$

where $M_{H_{Z}}$ is the total yawing moment about the hub. Equation 2 represents the instantaneous power required to drive the rotor at a constant angular velocity $\Omega$ averaged over one revolution. The effects of unsteadiness, compressibility, dynamic stall (if applicable), and the additional drag due to flap deflection is included in the calculation of $M_{H_{Z}}$. The engine must supply a torque equal to $-M_{H_{Z}}$ in order to maintain a constant angular velocity. The relation for $J_{P}$ is a general expression which is valid for rotor blades with or without ACF's.

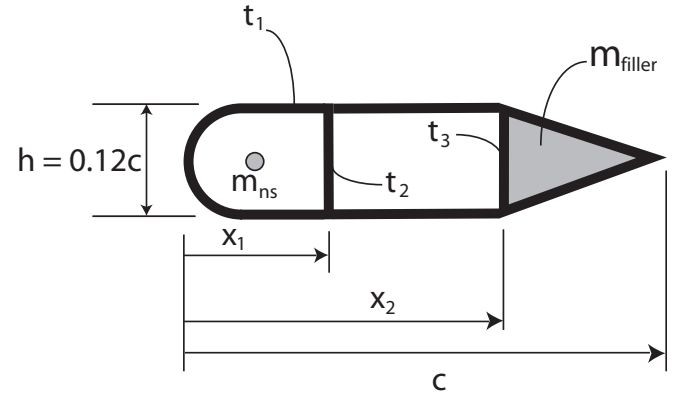

Figure 2. Simplified model of the blade structural member.
The vector of design variables $\mathbf{D}$ consists of the thicknesses $t_{1}, t_{2}, t_{3}$, and the non-structural mass $m_{n s}$ located at the shear center, as shown in Fig. 2. The three thickness design variables were defined as constant within 4 equally sized spanwise sections, while the non-structural mass design variable was defined as constant between the $68 \%$ and $100 \%$ blade stations. The $68 \%$ and $100 \%$ blade stations were chosen for the non-structural mass because previous studies have shown that non-structural masses are most effective for vibration reduction when they are distributed over the outboard $1 / 3$ of the blade. ${ }^{30,31}$ The nonstructural mass at the elastic axis inboard of the $68 \%$ station was set to zero. In addition, design variables representing the pretwist at the $50 \%$ and $100 \%$ stations $-\theta_{1}$ and $\theta_{2}$ respectively

- are included. The pretwist at the root is fixed at a baseline value $\theta_{0}$, and the twist is assumed to vary linearly between blade stations. Thus there are a total of 15 structural design variables in the passive optimization formulation. The design variables have side constraints to prevent them from reaching impractical values; these are stated as

$$
\mathbf{D}_{j}^{(L)} \leq \mathbf{D} \leq \mathbf{D}_{j}^{(U)}, \quad j=1,2, \ldots, N_{d v} .
$$

In addition, four types of behavior constraints, given by

$$
g_{i}(\mathbf{D}) \leq 0, \quad i=1,2, \ldots, N_{c},
$$

are placed on the design variables. The first type of behavior constraints are frequency placement constraints, which are prescribed upper and lower bounds on the fundamental flap, lag, and torsional frequencies of the blade. The frequency placement constraints on the fundamental flap frequency are written as

$$
\mathrm{g}_{\text {flap }}(\mathbf{D})=\frac{\omega_{F 1}}{\omega_{U}}-1 \leq 0
$$

and

$$
\mathrm{g}_{\text {flap }}(\mathbf{D})=1-\frac{\omega_{F 1}}{\omega_{L}} \leq 0
$$

where $\omega_{U}$ and $\omega_{L}$ are the prescribed upper and lower bounds on the fundamental flap frequency. Similar constraints are placed on the lag and torsional frequencies, i.e. glag and $g_{\text {torsion. }}$ In addition, all blade frequencies must differ from integer multiples of the angular velocity $-1 / \mathrm{rev}, 2 / \mathrm{rev}, 3 / \mathrm{rev}, \ldots$, etc. - to avoid undesirable resonances.

Another behavior constraint is an autorotational constraint, which ensures that mass redistributions produced during the optimization do not degrade the autorotational properties of the rotor. Several indices can be used to represent the autorotational properties of the blade; the one selected for this study is the requirement that the mass polar moment of inertia of the rotor be at least $80 \%$ of its baseline value, which implies:

$$
\mathrm{g}(\mathbf{D})=1-\frac{I_{P}}{0.8 I_{P 0}} \leq 0
$$

where $I_{P}$ is the mass polar moment of inertia of the rotor when it is spinning about the shaft, and $I_{P 0}$ is the baseline value. 
The third type of behavior constraints are aeroelastic stability margin constraints that can be stated as:

$$
\mathrm{g}_{k}(\mathbf{D})=\zeta_{k}+\left(\zeta_{k}\right)_{\min } \leq 0, \quad k=1,2, \ldots, N_{m}
$$

where $N_{m}$ is the number of normal modes, $\zeta_{k}$ is the real part of the hover eigenvalue for the $k^{t h}$ mode, and $\left(\zeta_{k}\right)_{\min }$ is the minimum acceptable damping level for the $k_{t h}$ mode. It should be noted that the most critical modes for stability are usually the first and second lag modes.

The final behavior constraint is a stress constraint obtained by substituting the blade stresses into Von Mises' criterion, which is expressed mathematically as

$$
\frac{2 \sigma_{x x}^{2}+6\left(\sigma_{x \eta}^{2}+\sigma_{x \zeta}^{2}\right)}{6}-\frac{\sigma_{\text {allowable }}^{2}}{3} \leq 0
$$

where $\sigma_{x x}, \sigma_{x \eta}$, and $\sigma_{x \zeta}$ are the axial and shear stresses, and $\sigma_{\text {allowable }}$ is the material yield stress divided by a factor of safety. At discrete values of the azimuth angle, Eq. 9 is evaluated at spanwise locations corresponding to the finite element nodes. The maximum evaluation of Eq. 9 is used for the constraint, and is given as

$$
\mathrm{g}(\mathbf{D})=\operatorname{MAX}\left[\frac{2 \sigma_{x x}^{2}+6\left(\sigma_{x \eta}^{2}+\sigma_{x \zeta}^{2}\right)}{6}-\frac{\sigma_{\text {allowable }}^{2}}{3}\right] \leq 0
$$

where MAX[ ] denotes the maximum value of Eq. 9 over each set of azimuth angle and blade stations at which it is evaluated. Therefore the stress constraint is enforced at the blade station and azimuth angle where the stress condition is most critical.

\section{III.B. Passive Reduction Approach}

To facilitate a global search of the design space in a reasonable amount of time, it is necessary to use global approximation, or surrogate methods, where the "true" objective function and expensive constraints are replaced with smooth functional relationships of acceptable accuracy that can be evaluated quickly. To construct the surrogates, the objective function and expensive constraints must first be evaluated over a set of design points. The surrogates are then generated by fitting the initial data points. In this process, function evaluations based on expensive helicopter simulations are needed to form the approximations. However, this initial investment of computer time is relatively small compared to global searches based on non-surrogate optimization methods. In this study, the objective functions given by Eqs. 1 and 2, and the stress constraint given by Eq. 10 are replaced by surrogates. The stress constraint is the only constraint which requires a forward flight simulation, and thus is computationally expensive enough to require its replacement by a surrogate.

Once the surrogates are obtained, optimal blade designs are found using the Efficient Global Optimization (EGO) algorithm. ${ }^{32}$ The EGO algorithm locates optimal designs while protecting against poor designs due to uncertainty, or error, in the approximate objective function's predictions. In EGO, a small number of initial design points are used to fit kriging approximations of the objective function and expensive constraints. Based on the stochastic nature of kriging, an expected improvement function (EIF) is formulated in order to facilitate the selection of additional sample points, known as infill samples, where expensive computer simulations are to be conducted. These sample points are chosen to be where there is a high probability of producing a superior design over the current best design and/or where the predictions of the surrogate objective function is unreliable due to a high degree of uncertainty. The EGO algorithm is depicted in Fig. 3. Additional details on the generation of the initial data set, the kriging interpolation, and the EIF formulation can be found in Ref. 13 .

In order to control the balance between protecting against errors in the surrogates while locating optimal designs based on the surrogate's predictions, an extension of the EIF known as the weighted expected improvement function (WEIF) is employed. ${ }^{33}$ The weighted expected improvement function (WEIF) is given by

$$
W \operatorname{EIF}(\mathbf{D})= \begin{cases}w \chi_{1}+(1-w) \chi_{2} & \text { if } s>0 \\ 0 & \text { if } s=0\end{cases}
$$

where the values of $\chi_{1}$ are large for designs the surrogate predicts to be superior to the current best design, $\chi_{2}$ is large for designs corresponding to a high degree of uncertainty in the surrogate's predictions, $s$ represents 


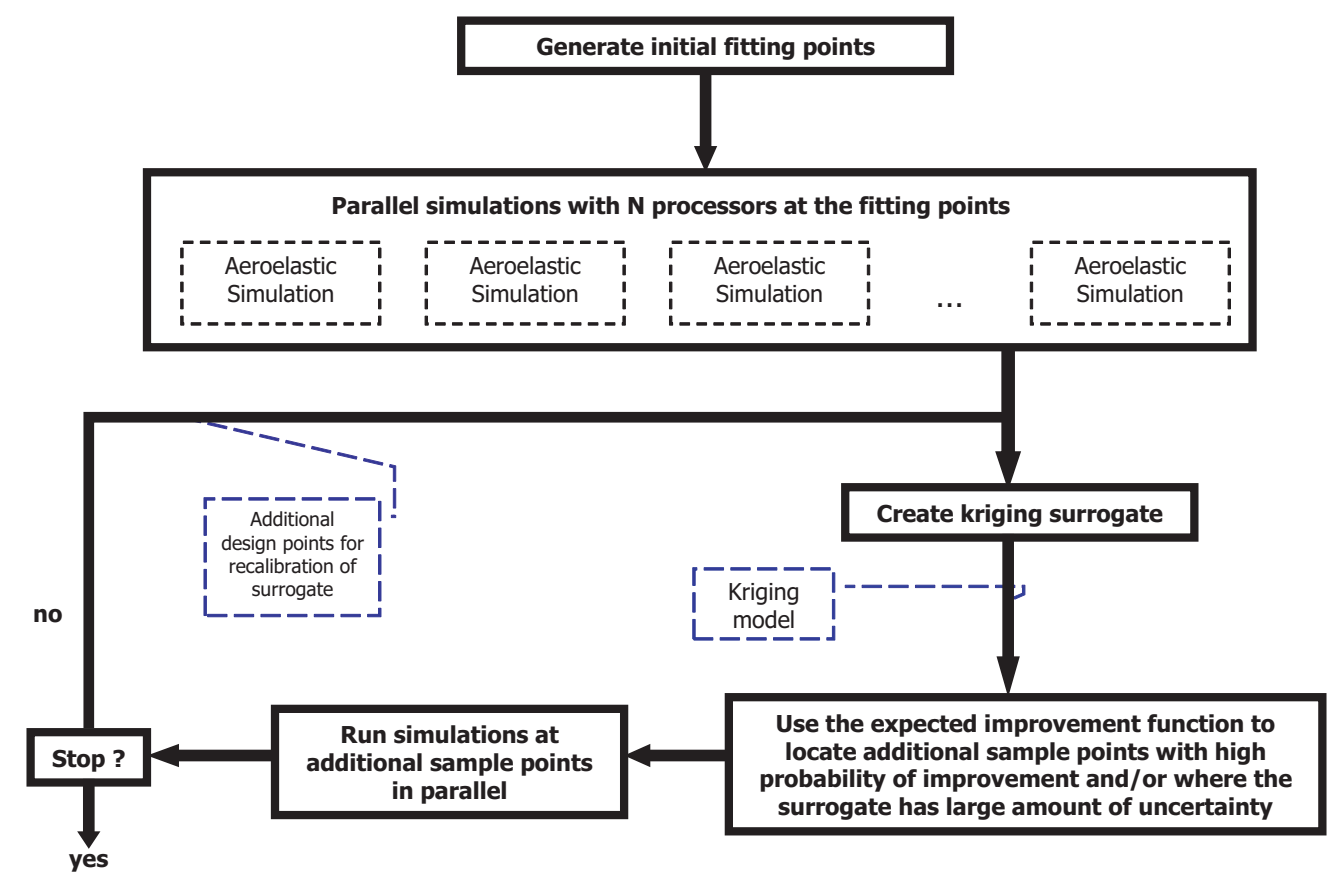

Figure 3. Efficient Global Optimization (EGO) algorithm.

the predicted error in the surrogate objective function, and $0 \leq w \leq 1$ is a user defined weight parameter. Expressions for $\chi_{1}, \chi_{2}$, and $s$ can be found in Ref. 13. Setting $w=0$ shifts the balance toward protecting against errors in the approximate objective function, while $w=1$ emphasizes the search for optimum designs obtained from surrogate based predictions. The blade designs corresponding to the infill sample points are obtained by maximizing the WEIF. In this implementation of EGO, a genetic algorithm from the iSIGHT software package ${ }^{34}$ is used to optimize the WEIF while enforcing the constraints on the blade design. A number of local optima of the WEIF equal to the number of available processors are selected as infill sample points in order to take advantage of parallel computation.

Since multiple objective functions are considered in this study, it is desirable to locate blade designs corresponding to the best trade-offs between the vibration and power objective functions. The best trade-off designs are known as Pareto optimal if no other designs can be found which correspond to superior objective function values for all objective functions. To locate a set of Pareto optimal designs, two approaches were employed to extend EGO for multi-objective function optimization. The first approach is a weighted sum approach in which both objective functions are combined into a single objective function, i.e.

$$
J_{\text {sum }}=W J_{V}+(1-W) J_{P} .
$$

Various values of $0 \leq W \leq 1$ are selected in order to convert the multi-objective function optimization problem into multiple single-objective function optimization problems. Thus, for each value of $W$, EGO is applied to $J_{\text {sum }}$.

In the second approach, EGO is modified to locate the Pareto designs associated with the expected improvement functions. The modified EGO algorithm proceeds as follows:

1. Weighted expected improvement functions corresponding to the vibration and power objective functions are generated from surrogates for each objective function.

2. Using the genetic algorithms available in iSIGHT, the Pareto optima of the two WEIF's are obtained.

3. Parallel simulations are conducted at each of the Pareto designs.

4. The additional design points are added to the fitting data used to create each surrogate objective function.

5. The surrogates corresponding to both objective functions are recalibrated with the updated fitting data. 
6. The process is repeated until the stopping condition is reached.

The best trade-off designs from both multi-objective function optimization approaches are combined into a data set which is filtered for the final Pareto optimal designs.

\section{III.C. Active Vibration and Power Reduction}

Active control of vibration and rotor power is based on the higher-harmonic control (HHC) algorithm. ${ }^{35}$ The stability, robustness, and convergence properties of the algorithm and a number of variants were explored in Ref. 36. The relaxed adaptive HHC variant detailed in Ref. 16 is employed in this study. The algorithm is based on a linear, frequency domain representation of helicopter response to control inputs. The input harmonics to the ACF consist of a combination of flap deflection angles having frequencies of 2, 3, 4 and $5 /$ rev. Thus, the total flap deflection is a combination of these contributions:

$$
\delta_{f}(\psi)=\sum_{N=2}^{5}\left[\delta_{N c} \cos (N \psi)+\delta_{N s} \sin (N \psi)\right]
$$

where $\delta_{N c}$ and $\delta_{N s}$ are the control amplitudes.

The control strategy is based on the minimization of a performance index which is a quadratic function of the quantities that are being reduced $\mathbf{z}_{k}$, and control input amplitudes $\mathbf{u}_{k}:{ }^{35,36}$

$$
J_{\mathrm{ACF}}\left(\mathbf{z}_{k}, \mathbf{u}_{k}\right)=\mathbf{z}_{k}^{\mathrm{T}} \mathbf{Q} \mathbf{z}_{k}+\mathbf{u}_{k}^{\mathrm{T}} \mathbf{W}_{\mathrm{u}} \mathbf{u}_{k} .
$$

The subscript $k$ refers to the $k^{\text {th }}$ control step, reflecting the discrete-time nature of the control. The optimal control inputs are given in Ref. 16. In the case of vibration reduction,

$$
\mathbf{z}_{k, \mathrm{VR}}=\left[\begin{array}{llllll}
F_{4 X} & F_{4 Y} & F_{4 Z} & M_{4 X} & M_{4 Y} & M_{4 Z}
\end{array}\right]^{\mathrm{T}} .
$$

For rotor power reduction,

$$
\mathbf{z}_{k, \mathrm{PR}}=\left[J_{P}\right] .
$$

The vector $\mathbf{z}_{k}$ is obtained by combining $\mathbf{z}_{k, \mathrm{VR}}$ and $\mathbf{z}_{k, \mathrm{PR}}$ as

$$
\mathbf{z}_{k}=\left[\frac{\mathbf{z}_{k, \mathrm{VR}}}{\mathbf{z}_{k, \mathrm{PR}}}\right],
$$

In Eq. 14, $\mathbf{Q}$ is a diagonal weighting matrix and the $\mathbf{W}_{\mathbf{u}}$ matrix is used to enforce saturation limits on the flap deflections of $\delta_{\text {fmax }} \leq 4^{\circ}$. The weight matrices associated with vibration and power reduction, $\mathbf{Q}_{\mathrm{VR}}$ and $\mathbf{Q}_{\mathrm{PR}}$, are combined as

$$
\mathbf{Q}=\left[\begin{array}{cc}
\left(W_{\alpha}\right) \cdot\left[\mathbf{Q}_{\mathrm{VR}}\right] & 0 \\
0 & \left(1-W_{\alpha}\right) \cdot\left[\mathbf{Q}_{\mathrm{PR}}\right]
\end{array}\right],
$$

where $0 \leq W_{\alpha} \leq 1$.

\section{III.D. Sequential Active/Passive Approach}

The sequential approach is implemented in two steps - (1) first, the blade is structurally optimized using the EGO algorithm, and (2) a partial span actively controlled trailing edge flap is added to the optimized design and the controller, operating in a closed-loop mode, determines the flap deflections required for vibration reduction, power reduction, or simultaneous vibration and power reduction.

\section{III.E. Combined Active/Passive Approach}

In this study, the combined active/passive approach employed in Ref. 17 is extended for application to multiobjective function optimization. For vibration reduction, the approach based on Ref. 17 is characterized by the following steps:

1. The optimizer selects a blade design characterized by the structural design variables. 
2. For the given blade design, the active control algorithm determines the optimal control input for vibration reduction based on Eq. 14.

3. The passive vibration objective function, Eq. 1, is evaluated for the blade design and control input.

4. The resulting value of the objective function is returned to the optimizer.

The optimum design returned by the combined approach outlined above would correspond to the minimum vibration levels when utilizing active control. For power reduction, the vibration objective function would be replaced by the performance objective function given by Eq. 2, and $W_{\alpha}$ would be set to 0 .

Since vibration reduction was the only objective considered in Ref. 17, the control algorithm always selected the optimal control inputs for maximum vibration reduction; i.e. $W_{\alpha}=1$. However, in this study it is necessary to select the blade designs and the corresponding control settings which result in the best trade-offs between vibration and power reduction. Therefore, $W_{\alpha}$ is treated as an additional design variable, and each iteration of the combined active/passive approach employed in this study proceeds as follows:

1. For a given blade design and $W_{\alpha}$ setting, the active control algorithm determines the optimal control input based on Eq. 14.

2. The vibration and performance objective functions are evaluated for the blade designs and control settings.

3. Using the multi-objective function optimization approaches based on the EGO algorithm, potential Pareto optimal active/passive configurations characterized by the 15 structural design variables and the additional design variable $W_{\alpha}$ are selected.

4. The process is repeated until the stopping condition is reached.

\section{Results}

Vibration and power reduction results using the EGO and active control algorithms are presented in this section. Parameters for the helicopter simulations are given first, followed by optimization results.

\section{IV.A. Helicopter Parameters}

The helicopter configuration and flight condition parameters used to generate all of the results in this section are given in Table 1. The simulations are conducted at a level flight condition with an advance ratio of 0.35 . High vibration levels due to dynamic stall are encountered at this flight condition. Figure 4 illustrates the geometrical data needed for the propulsive trim calculation which is used in this study.

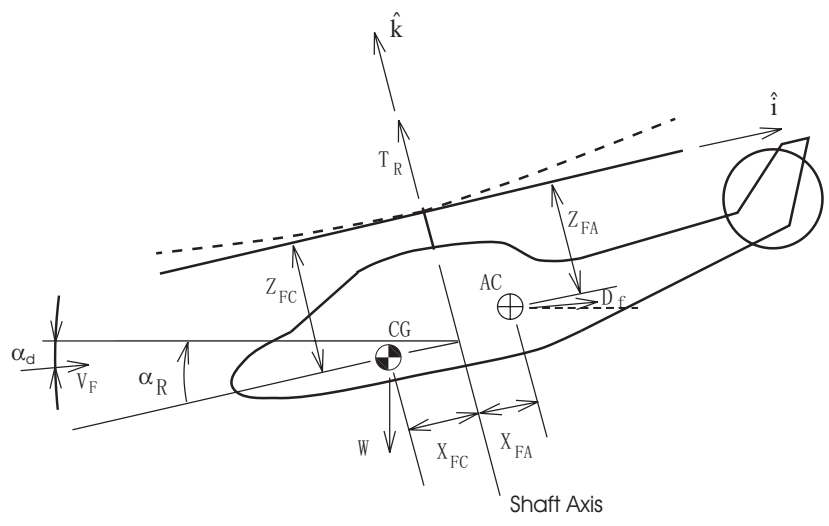

Figure 4. Helicopter in forward flight.

In addition to the data in Table 1, additional information is required to represent the constant crosssectional parameters, material properties, objective function, constraints, and the finite element discretization of the blade. Part of this information is given in Table 2, and the rest of the required data is provided in the text. 
Table 1. Rotor and helicopter parameters

\begin{tabular}{ll}
\hline \hline$\underline{\text { Rotor Data }}$ & \\
$R=4.91 \mathrm{~m}$ & $\Omega=425 \mathrm{RPM}$ \\
$N_{b}=4$ & $c=0.05498 R$ \\
$\beta_{p}=2.5^{\circ}$ & $C_{d o}=0.01$ \\
$\theta_{0}=5.6^{\circ}$ & $C_{W}=0.005$ \\
$\sigma=0.07$ & $C_{T} / \sigma=0.0714$ \\
$X_{F A}=0.0$ & $Z_{F A}=0.3$ \\
$X_{F C}=0.0$ & $Z_{F C}=0.3$ \\
$C_{d f}=0.01$ & $m_{0}=5.57 \mathrm{~kg} / \mathrm{m}$ \\
& \\
Flight Condition & \\
$\mu=0.35$ & $\alpha_{\mathrm{d}}=0^{\circ}$ \\
MBB BO-105 baseline blade & \\
$\omega_{L 1}=0.729$ & $\omega_{F 1}=1.125$ \\
$\omega_{T 1}=3.263$ & \\
$\theta_{1}=1.6^{\circ}$ & $\theta_{2}=-2.4^{\circ}$ \\
\hline \hline
\end{tabular}

The weighting factors in Eq. $1, K_{S}$ and $K_{M}$, are selected to be 1 . These weights result in a vibration objective function which represents the sum of the $4 / \mathrm{rev}$ oscillatory hub shear resultant and the $4 / \mathrm{rev}$ oscillatory hub moment resultant in the hub-fixed non-rotating frame.

The following side constraints are enforced:

$$
\begin{gathered}
1.0 \mathrm{~mm} \leq t_{1} \leq 8.0 \mathrm{~mm} \\
1.0 \mathrm{~mm} \leq t_{2}, t_{3} \leq 12.0 \mathrm{~mm} \\
0.0 \leq m_{n s} / m_{0} \leq 0.25 \\
-4^{\circ} \leq \theta_{1}, \theta_{2} \leq 4^{\circ}
\end{gathered}
$$

The upper and lower bounds used for the frequency placement constraints, /rev, are given below.

$$
\begin{aligned}
& 0.50 \leq \omega_{L 1} \leq 0.80 \\
& 1.05 \leq \omega_{F 1} \leq 1.20 \\
& 2.50 \leq \omega_{T 1} \leq 6.50
\end{aligned}
$$

For the aeroelastic stability constraints given by Eq. 8, the minimum acceptable damping for all modes, $\left(\zeta_{k}\right)_{\min }$, is chosen to be $0.01 .{ }^{21,24}$ Additionally, the constraints are modified for the $2^{\text {nd }}$ lag mode, which can sometimes be slightly unstable. To prevent this situation, a small amount of structural damping is added to this mode. For this study, $0.5 \%$ structural damping is added to stabilize the $2^{\text {nd }}$ lag mode of the baseline blade. A factor of safety of 1.5 is used for the stress constraint. The rotor blade was discretized into the 6 finite elements shown in Fig. 5. 
Table 2. Fixed parameters defining the structure and cross section

\begin{tabular}{l}
\hline \hline$\frac{\text { Aluminum Material Properties }}{E=70.7 \mathrm{GPa}}$ \\
$\nu=0.33$ \\
$\rho_{\text {struct }}=2700 \mathrm{~kg} / \mathrm{m}^{3}$ \\
$\sigma_{Y}=324 \mathrm{MPa}$ \\
Non-structural Filler Mass Density \\
$\rho_{\text {filler }}=237.4 \mathrm{~kg} / \mathrm{m}^{3}$ \\
$\underline{\text { Locations of the Vertical Walls }}$ \\
$x_{1}=65.4 \mathrm{~mm}$
\end{tabular}

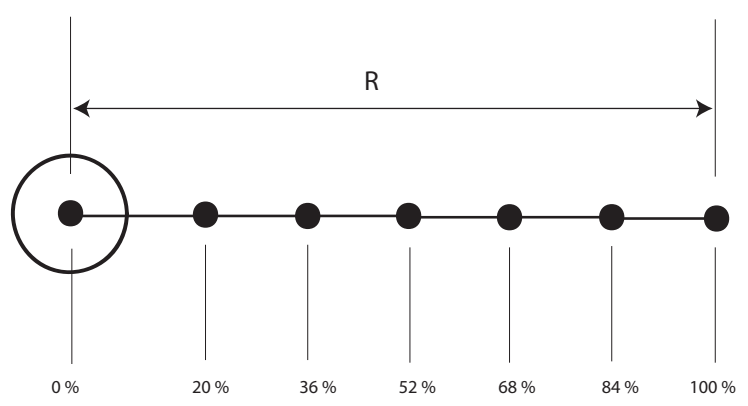

Figure 5. Finite element node locations.

\section{IV.B. Passive Reduction Results}

Efficient Global Optimization results using the weighted expected improvement function are given in this section. The EGO algorithm was initialized with 200 data points and the initial iterations were run with a weight setting of $w=0.2$. Once EGO failed to improve upon the best design from the previous iteration, the weight setting was changed to $w=0.8$. The EGO algorithm was stopped once the iterations with $w=0.8$ failed to improve upon the best design from the previous iteration. This weight scheme was selected for two reasons: (1) so that the EGO algorithm would focus on a more global search initially and then move to a more local search within regions of high probability of improvement, and (2) because these weights were shown to be effective for BVI vibration reduction. ${ }^{37}$ The weighted sum approach to multi-objective function optimization was conducted with the following values of $W: 0.0,0.25,0.50,0.75$ and 1.0. All optimization results are compared to a baseline blade with cross-sectional properties resembling an MBB BO-105 blade.

The amount of vibration and power reduction for the best trade-off, or Pareto optimal designs are given in Table 3 along with the fundamental frequencies and pretwist design variables which characterize the blade designs. The best vibration design corresponds to $34.2 \%$ reduction of the vibration objective function and a $13.9 \%$ increase in power consumption, while the best power design corresponds to a $30.4 \%$ increase in vibration levels and $3.30 \%$ performance enhancement relative to the baseline blade. These results demonstrate that there are strong trade-offs between the two objectives at this flight condition. Despite the strong trade-offs, the multi-objective function optimization approach based on EGO led to a design corresponding to $9.8 \%$ vibration reduction and $2.64 \%$ performance enhancement. A trade-off design such as P2, which corresponds to simultaneous reduction of the two objectives, would not have been located without employing multi-objective function optimization techniques.

The vibratory loads corresponding to the best vibration and power designs, as well as the trade-off design P2, are shown in Fig. 6. It is clear from Fig. 6 that the three hub shears are the dominant components of the overall vibration objective function. The best vibration design reduces the overall objective function by decreasing $F_{4 X}$ and $F_{4 Y}$ by $48 \%$ and $32 \%$ respectively. In contrast, there is little difference between the baseline values of $F_{4 X}$ and $F_{4 Y}$ and those corresponding to P2. For P2, the overall vibration objective 
Table 3. Best trade-off designs

\begin{tabular}{cccccccc}
\hline \hline $\begin{array}{c}\text { Design } \\
\text { Identifier }\end{array}$ & $\begin{array}{c}\text { Vibration } \\
\text { Reduction }\end{array}$ & $\begin{array}{c}\text { Power } \\
\text { Reduction }\end{array}$ & $\begin{array}{c}\omega_{L 1} \\
(/ \mathrm{rev})\end{array}$ & $\begin{array}{c}\omega_{F 1} \\
(/ \mathrm{rev})\end{array}$ & $\begin{array}{c}\omega_{T 1} \\
(/ \mathrm{rev})\end{array}$ & $\theta_{1}$ & $\theta_{2}$ \\
\hline P1 & $34.24 \%$ & $-13.91 \%$ & 0.776 & 1.074 & 5.997 & $-2.36^{\circ}$ & $1.15^{\circ}$ \\
P2 & $9.82 \%$ & $2.64 \%$ & 0.589 & 1.052 & 4.163 & $3.62^{\circ}$ & $-2.36^{\circ}$ \\
P3 & $-27.00 \%$ & $2.95 \%$ & 0.705 & 1.068 & 5.503 & $3.24^{\circ}$ & $-0.41^{\circ}$ \\
P4 & $-30.06 \%$ & $3.08 \%$ & 0.658 & 1.062 & 5.548 & $4.00^{\circ}$ & $-0.98^{\circ}$ \\
P5 & $-30.36 \%$ & $3.26 \%$ & 0.657 & 1.062 & 5.530 & $4.00^{\circ}$ & $-0.91^{\circ}$ \\
P6 & $-30.43 \%$ & $3.30 \%$ & 0.655 & 1.061 & 5.540 & $4.00^{\circ}$ & $-0.91^{\circ}$ \\
\hline \hline
\end{tabular}

function is reduced due to the $20 \%$ decrease of the vertical shear $F_{4 Z}$. In the case of the best power design, the $23-64 \%$ increase in the hub shears along with the $277 \%$ increase in $M_{4 Z}$ result in the significant vibration penalty associated with optimization for power reduction.

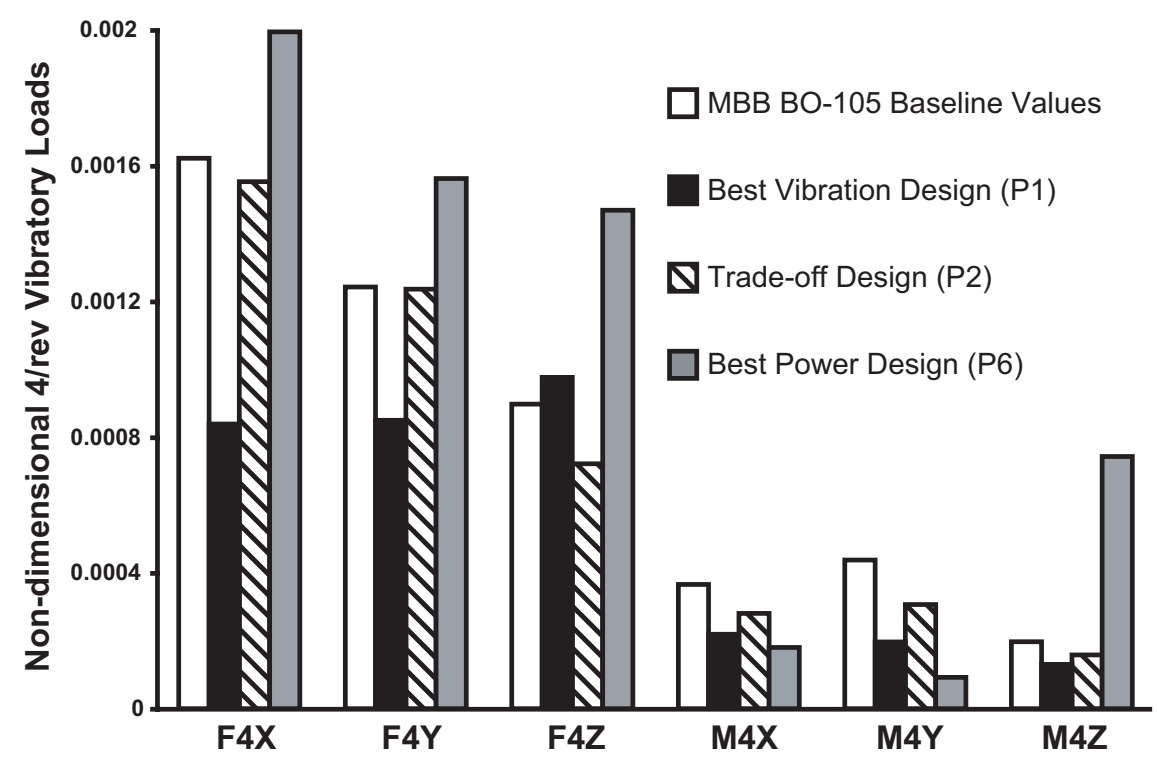

Figure 6. Vibratory loads corresponding to the passively optimized designs.

\section{IV.C. Sequential Active/Passive Reduction Results}

The active/passive results were generated by adding a single plain flap, as depicted in Fig. 1, to the six trade-off designs. The flap is centered at the $75 \%$ blade station and has a total spanwise dimension of $0.12 R$ and a chordwise dimension of 0.20c. The diagonal components of $\mathbf{Q}_{\mathrm{VR}}$ and $\mathbf{Q}_{\mathrm{PR}}$ were set to 1. For each of the Pareto optimal designs, active control was implemented for $W_{\alpha}$ settings of 0.0, 0.25, 0.50, 0.75 and 1.0. Thus, there were a total of 30 active/passive configurations in the sequential approach.

The results associated with the best trade-offs from the 30 active/passive configurations are given in Table 4. Compared to the best vibration design from passive optimization, up to $22.8 \%$ additional vibration reduction was obtained by using active control. However, even when augmenting P1 with active control, the high power consumption could not be significantly reduced and remained substantially higher than the baseline value. By augmenting P5 with active control, up to $0.25 \%$ additional power reduction was achieved compared to the best power design without active control. However, the P5 active/passive configurations correspond to a $27.3-56.0 \%$ increase in vibration levels.

Although the performance characteristics of the best vibration design could not be significantly augmented by using active control, and vice versa, the sequential approach resulted in increased levels of simultaneous reduction with $\mathrm{P} 2$. The active/passive $\mathrm{P} 2$ configurations with $W_{\alpha}$ settings of $1.0,0.5$, and 0.25 , resulted in 
Table 4. Best trade-off configurations obtained from the sequential approach

\begin{tabular}{cccc}
\hline \hline Design Identifier & $W_{\alpha}$ & Vibration Reduction & Power Reduction \\
\hline P1 & 1.00 & $57.07 \%$ & $-14.12 \%$ \\
P1 & 0.75 & $51.37 \%$ & $-14.07 \%$ \\
P1 & 0.50 & $50.20 \%$ & $-13.77 \%$ \\
P1 & 0.25 & $41.24 \%$ & $-13.64 \%$ \\
P2 & 1.00 & $27.62 \%$ & $3.06 \%$ \\
P2 & 0.50 & $27.45 \%$ & $3.09 \%$ \\
P2 & 0.25 & $27.35 \%$ & $3.13 \%$ \\
P2 & 0.00 & $0.76 \%$ & $3.24 \%$ \\
P5 & 0.25 & $-27.33 \%$ & $3.39 \%$ \\
P5 & 0.00 & $-56.04 \%$ & $3.55 \%$ \\
\hline \hline
\end{tabular}

an additional $17.5-17.8 \%$ vibration reduction and $0.42-0.49 \%$ performance enhancement. Note that P2 with $W_{\alpha}=0.75$ was not among the best trade-offs because setting $W_{\alpha}=1.0$ resulted in lower vibration and power levels. Although a setting of $W_{\alpha}=0.75$ emphasizes rotor power reduction compared to $W_{\alpha}=1.0$, the control algorithm may not always result in the optimal control input. ${ }^{16}$

\section{IV.D. Combined Active/Passive Reduction Results}

The best trade-off configurations from the combined active/passive approach are given in Table 5. In addition to the total amounts of vibration and power reduction obtained with active control, the uncontrolled levels are also provided. Note that C5 corresponds to $5.93 \%$ uncontrolled power reduction, which is superior to P6. Therefore, a design that should have been located with the passive approach was missed. This indicates that conducting additional EGO iterations in the passive approach would lead to improved designs.

Table 5. Active/passive results associated with the combined approach

\begin{tabular}{|c|c|c|c|c|c|c|c|c|c|c|}
\hline $\begin{array}{l}\text { Design } \\
\text { Identifier }\end{array}$ & $W_{\alpha}$ & $\begin{array}{l}\text { Vibration } \\
\text { Reduction } \\
\text { (controlled) }\end{array}$ & $\begin{array}{c}\text { Power } \\
\text { Reduction } \\
\text { (controlled) }\end{array}$ & $\begin{array}{c}\text { Vibration } \\
\text { Reduction } \\
\text { (uncontrolled) }\end{array}$ & $\begin{array}{c}\text { Power } \\
\text { Reduction } \\
\text { (uncontrolled) }\end{array}$ & $\begin{array}{c}\omega_{L 1} \\
(/ \mathrm{rev})\end{array}$ & $\begin{array}{c}\omega_{F 1} \\
(/ \text { rev })\end{array}$ & $\begin{array}{c}\omega_{T 1} \\
(/ \mathrm{rev})\end{array}$ & $\theta_{1}$ & $\theta_{2}$ \\
\hline $\mathrm{C} 1$ & 0.51 & $25.55 \%$ & $-0.96 \%$ & $-4.34 \%$ & $-0.53 \%$ & 0.782 & 1.077 & 5.568 & $-1.61^{\circ}$ & $-3.06^{\circ}$ \\
\hline $\mathrm{C} 2$ & 0.25 & $19.10 \%$ & $1.46 \%$ & $2.30 \%$ & $1.61 \%$ & 0.747 & 1.070 & 5.998 & $2.68^{\circ}$ & $1.48^{\circ}$ \\
\hline C3 & 0.75 & $16.49 \%$ & $2.70 \%$ & $-6.02 \%$ & $1.76 \%$ & 0.603 & 1.053 & 4.279 & $1.73^{\circ}$ & $-2.80^{\circ}$ \\
\hline $\mathrm{C} 4$ & 0.60 & $-11.86 \%$ & $3.17 \%$ & $-44.36 \%$ & $3.59 \%$ & 0.652 & 1.062 & 5.539 & $3.52^{\circ}$ & $0.18^{\circ}$ \\
\hline C5 & 0.45 & $-53.04 \%$ & $5.97 \%$ & $-81.35 \%$ & $5.93 \%$ & 0.570 & 1.054 & 4.852 & $3.43^{\circ}$ & $2.17^{\circ}$ \\
\hline
\end{tabular}

Other than C5, the designs in Table 5 are inferior in terms of vibration and power compared to at least one of the configurations found from the sequential approach. Therefore, as in the case of the passive approach, additional iterations with the combined approach were necessary to locate the best designs. Since it was apparent that optimal active/passive configurations were missed, $W_{\alpha}$ settings of $0.0,0.25,0.50,0.75$ and 1.0 were considered for each of the blade designs corresponding to $\mathrm{C} 1-\mathrm{C} 5$. This is equivalent to conducting a local search of the design space in the vicinity of the best designs returned from a global search.

The resulting 25 active/passive configurations from the combined approach were compared to those from the sequential approach and the Pareto optimal configurations were identified. These results are provided in Table 6. The presence of $\mathrm{C} 1, \mathrm{C} 2, \mathrm{C} 4$, and $\mathrm{C} 5$ among the best trade-offs in Table 6 demonstrates that the combined approach led to useful configurations which would have been overlooked if only sequential optimization were employed. For instance, $\mathrm{C} 2$ corresponds to a $2.30 \%$ and $1.61 \%$ reduction in vibration and power respectively without the benefits of active control. By comparison, P2 corresponds to $9.82 \%$ and $2.64 \%$ reductions in vibration and power respectively, and thus is superior to $\mathrm{C} 2$ in terms of passive characteristics. Therefore, when employing the sequential approach, $\mathrm{C} 2$ would have been eliminated because 
it is not Pareto optimal in terms of the passive vibration and power objectives. However, when using active control with $W_{\alpha}=1$, an additional $34.2 \%$ vibration reduction relative to the uncontrolled design is obtained, compared to $17.8 \%$ with P2. Thus, C2 is superior to P2 in terms of the total amount of vibration reduction which can be achieved with active control.

Table 6. Best trade-off configurations obtained from both active/passive approaches

\begin{tabular}{cccc}
\hline \hline Design Identifier & $W_{\alpha}$ & Vibration Reduction & Power Reduction \\
\hline P1 & 1.00 & $57.07 \%$ & $-14.12 \%$ \\
P1 & 0.75 & $51.37 \%$ & $-14.07 \%$ \\
P1 & 0.50 & $50.20 \%$ & $-13.77 \%$ \\
P1 & 0.25 & $41.24 \%$ & $-13.64 \%$ \\
C1 & 1.00 & $36.85 \%$ & $-1.40 \%$ \\
C2 & 1.00 & $36.50 \%$ & $1.17 \%$ \\
C2 & 0.75 & $30.49 \%$ & $1.28 \%$ \\
P2 & 1.00 & $27.62 \%$ & $3.06 \%$ \\
P2 & 0.50 & $27.45 \%$ & $3.09 \%$ \\
P2 & 0.25 & $27.35 \%$ & $3.13 \%$ \\
P2 & 0.00 & $0.76 \%$ & $3.24 \%$ \\
C4 & 0.50 & $-24.65 \%$ & $3.36 \%$ \\
P5 & 0.25 & $-27.33 \%$ & $3.39 \%$ \\
C4 & 0.25 & $-32.98 \%$ & $3.49 \%$ \\
C5 & 0.75 & $-52.03 \%$ & $5.94 \%$ \\
C5 & 0.50 & $-52.99 \%$ & $5.99 \%$ \\
C5 & 0.00 & $-83.68 \%$ & $6.09 \%$ \\
\hline \hline
\end{tabular}

The active/passive configurations from the sequential and combined approaches, along with the active control results corresponding to the baseline design with $W_{\alpha}$ settings of $0.0,0.25,0.50,0.75$ and 1.0 , are plotted in the objective function space in Fig. 7. The Pareto front is obtained by connecting each of the best trade-off points in Table 6. It is clear from Fig. 7 that the trade-offs obtained with the baseline design are inferior to those from the active/passive optimization approaches.

The following design options are apparent when moving from left to right on the Pareto front:

1. The designer can select configuration C5, which would result in 5.94-6.09\% performance enhancement. However, there is a $52-84 \%$ increase in vibration levels associated with the lowest power option.

2. Significant levels of vibration reduction and performance enhancement can be obtained by selecting the $\mathrm{C} 2$ or P2 trade-off designs. While the Pareto optimal active/passive configurations based on C2 correspond to $2.9-6.0 \%$ more vibration reduction than P2, P2 results in $1.8-2.1 \%$ less power consumption. Note that if P2 is selected, it is unlikely that the pilot would set $W_{\alpha}$ to 0 since this would result in only an additional $0.11 \%$ power reduction at the expense of a $26.6 \%$ increase in vibration compared to using $W_{\alpha}=0.25$.

3. The best vibration option would be to select $\mathrm{P} 1$, which would result in $41.2-57.1 \%$ vibration reduction. However, the $13.6-14.1 \%$ degradation in performance compared to the baseline design would substantially increase the cost of implementing such a design.

\section{Conclusions}

The results show that actively controlled flaps can be used to enhance vibration and performance characteristics of structurally optimized blades at high advance ratios. Since strong trade-offs between the vibration and power objective functions were observed, a multi-objective function optimization approach was required to identify a structurally optimized design corresponding to simultaneous vibration and power reduction. 


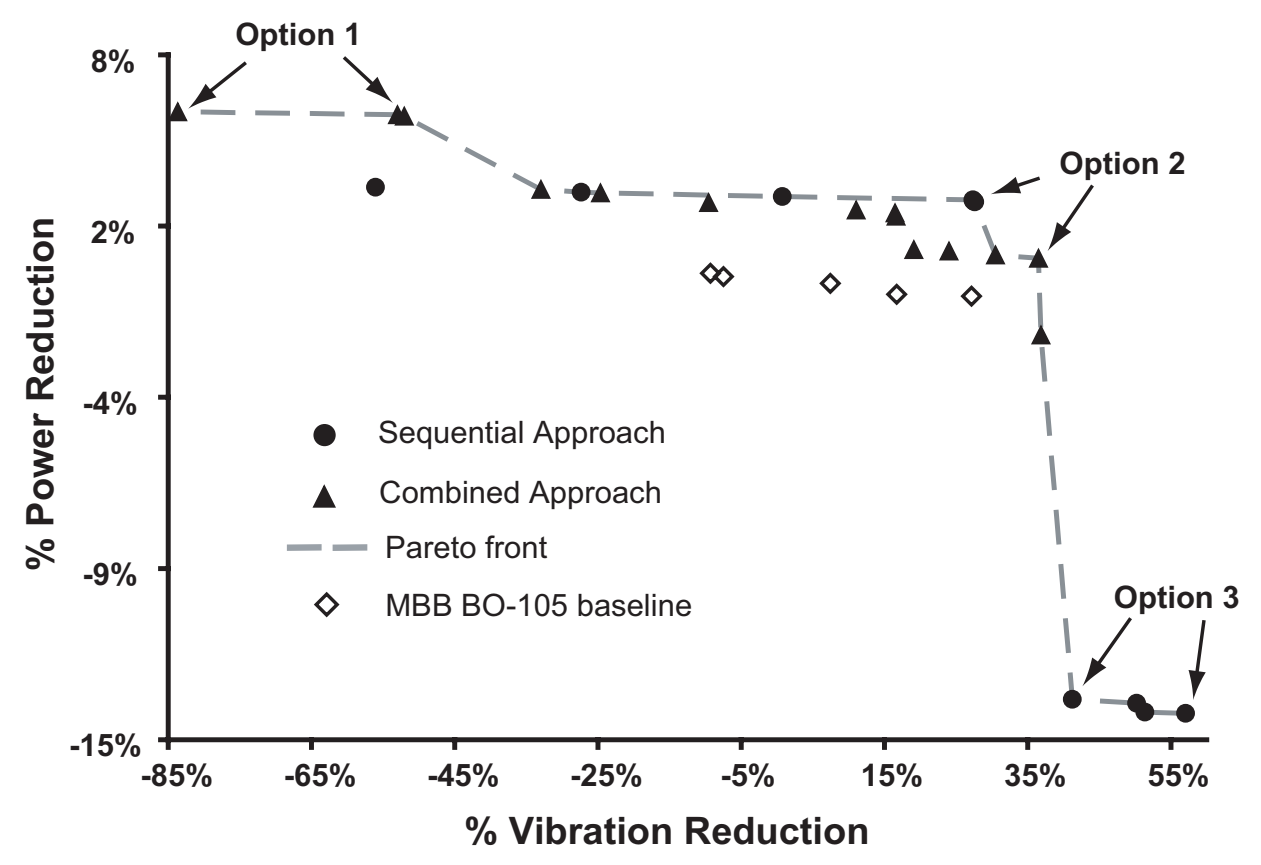

Figure 7. Pareto front.

Similarly, the best active/passive configuration for vibration reduction differed from that for power reduction. Therefore, the best set of trade-offs among the active/passive designs was identified using sequential and combined approaches. Both active/passive optimization approaches led to useful designs, even though the results indicate that better designs could be located with additional iterations. The principal results from this study are summarized below.

1. The best vibration design using structural optimization based on the EGO algorithm corresponded to $34.2 \%$ vibration reduction and a $13.9 \%$ increase in required rotor power.

2. The best design in terms of power consumption corresponded to $5.93 \%$ power reduction and a $81.4 \%$ increase in vibration levels.

3. Among the best active/passive configurations, the best design for vibration reduction corresponded to $41.2-57.1 \%$ vibration reduction, and a $13.6-14.1 \%$ performance degradation. Similarly, the best active/passive option for performance enhancement corresponded to $5.94-6.09 \%$ power reduction, and $52.0-83.7 \%$ increases in vibration levels.

4. Two designs corresponding to significant levels of vibration and power reduction were identified using the sequential and combined approaches: the first decreases vibration and power levels by $30.5-36.5 \%$ and $1.2-1.3 \%$ respectively, and the second corresponds to $27.4-27.6 \%$ vibration reduction and $3.06-3.13 \%$ performance enhancement.

\section{Acknowledgments}

This research was supported in part by a NASA Graduate Student Research Fellowship for B. Glaz with Dr. W. Warmbrodt as grant monitor. Partial support from the Center for Rotorcraft Innovation under WBS 2007-B-01-01.2-A17, as well as the Vertical Lift Research Center of Excellence (VLRCOE) sponsored by the NRTC with Dr. M. Rutkowski as grant monitor is also hereby acknowledged.

\section{References}

\footnotetext{
${ }^{1}$ Friedmann, P. P., "Helicopter Vibration Reduction Using Structural Optimization with Aeroelastic/Multidisciplinary Constraints - A Survey," Journal of Aircraft, Vol. 28, No. 1, January 1991, pp. 8-21.
} 
${ }^{2}$ Celi, R., "Recent Applications of Design Optimization to Rotorcraft-A Survey," Journal of Aircraft, Vol. 36, No. 1, January-February 1999, pp. 176-189.

${ }^{3}$ Ganguli, R., "Survey of Recent Developments in Rotorcraft Design Optimization," Journal of Aircraft, Vol. 41, No. 3, May-June 2004, pp. 493-510.

${ }^{4}$ Friedmann, P. P. and Millott, T. A., "Vibration Reduction in Rotorcraft Using Active Control: A Comparison of Various Approaches," Journal of Guidance, Control, and Dynamics, Vol. 18, No. 4, July-August 1995, pp. 664-673.

${ }^{5} \mathrm{He}$, C. J. and Peters, D. A., "Optimization of Rotor Blades for Combined Structural, Dynamic, and Aerodynamic Properties," Structural Optimization, Vol. 5, 1992, pp. $37-44$.

${ }^{6}$ Walsh, J. L., LaMarsh II, W. J., and Adelman, H. M., "Fully Integrated Aerodynamic/Dynamic Optimization of Helicopter Rotor Blades," Mathematical and Computer Modelling, Vol. 18, No. 3/4, 1993, pp. 53 - 72.

${ }^{7}$ Crossley, W. A., "Genetic Algorithm Approaches for Multiobjective Design of Rotor Systems," 6th AIAA, NASA, and ISSMO Symposium on Multidisciplinary Analysis and Optimization, Bellevue, WA, Sept. 4 - 6 1996, pp. 384 - 394, AIAA Paper 1996-4025.

${ }^{8}$ Zhao, Q. and Xu, G., "A Study on Aerodynamic and Acoustic Characteristics of Advanced Tip-Shape Rotors," Journal of the American Helicopter Society, Vol. 52, No. 3, July 2007, pp. $201-213$.

${ }^{9}$ Collins, K., Bain, J., Sankar, L., Egolf, T. A., Janakiram, R. D., Brentner, K., and Lopes, L., "Pareto Frontier Method for Multi-disciplinary Optimization of Helicopter Rotors," American Helicopter Society's Specialist's Conference on Aeromechanics, San Francisco, CA, Jan. 23 - 252008.

${ }^{10}$ Queipo, N. V., Haftka, R. T., Shyy, W., Goel, T., Vaidyanathan, R., and Tucker, P. K., "Surrogate-Based Analysis and Optimization," Progress in Aerospace Sciences, Vol. 41, 2005, pp. 1-28.

${ }^{11}$ Glaz, B., Friedmann, P. P., and Liu, L., "Surrogate Based Optimization of Helicopter Rotor Blades for Vibration Reduction in Forward Flight," Structural and Multidisciplinary Optimization, Vol. 35, No. 4, April 2008, pp. 341 - 363.

${ }^{12}$ Glaz, B., Goel, T., Liu, L., Friedmann, P. P., and Haftka, R. T., "Application of a Weighted Average Surrogate Approach to Helicopter Rotor Blade Vibration Reduction," 48th AIAA/ASME/ASCE/AHS/ACS Structures, Structural Dynamics and Materials Conference, Honolulu, HI, April 23-26 2007, pp. 1-25, AIAA Paper 2007-1898.

${ }^{13}$ Glaz, B., Friedmann, P. P., and Liu, L., "Helicopter Vibration Reduction Throughout the Entire Flight Envelope Using Surrogate Based Optimization," 63rd Annual Forum of the American Helicopter Society, Vol. II, Virginia Beach, VA, May 1-3 2007, pp. 1277-1292.

${ }^{14}$ Patt, D., Liu, L., and Friedmann, P. P., "Rotorcraft Vibration Reduction and Noise Prediction Using a Unified Aeroelastic Response Simulation," Journal of the American Helicopter Society, Vol. 50, No. 1, January 2005, pp. 95-106.

${ }^{15}$ Depailler, G. and Friedmann, P. P., "Alleviation of Dynamic Stall Induced Vibrations Using Actively Controlled Flaps," 58th Annual Forum of the American Helicopter Society, Montreal, Canada, June 2002.

${ }^{16}$ Liu, L., Friedmann, P. P., Kim, I., and Bernstein, D. S., "Vibration Reduction and Performance Enhancement in Rotorcraft Using Active Flaps at High Advance Ratios," 47th AIAA/ASME/ASCHE/AHS/ASC Structures, Structural Dynamics \& Materials Conference, Newport, RI, May 1-4 2006, pp. 1-21, AIAA Paper 2006-1861.

${ }^{17}$ Zhang, J., Smith, E. C., and Wang, K. W., "Active-Passive Hybrid Optimization of Rotor Blades with Trailing Edge Flaps," Journal of the American Helicopter Society, Vol. 49, No. 1, January 2004, pp. 54-65.

${ }^{18}$ Zhang, J., Active-Passive Hybrid Optimization of Rotor Blades With Trailing Edge Flaps, Ph.D. thesis, The Pennsylvania State University, 2001.

${ }^{19}$ Glaz, B., Friedmann, P. P., and Liu, L., "Vibration and Noise Reduction of Helicopter Rotors Using an Active/Passive Approach," American Helicopter Society's Specialist's Conference on Aeromechanics, San Francisco, CA, Jan. 23 - 252008.

${ }^{20}$ Vanderplaats, G. N., CONMIN - A Fortran Program for Constrained Function Minimization, NASA TM-X 62282, Aug. 1973.

${ }^{21}$ Yuan, K. A. and Friedmann, P. P., "Structural Optimization for Vibratory Loads Reduction of Composite Helicopter Rotor Blades with Advanced Geometry Tips," Journal of the American Helicopter Society, Vol. 43, No. 3, July 1998, pp. 246256.

${ }^{22}$ Myrtle, T. F. and Friedmann, P. P., "Application of a New Compressible Time Domain Aerodynamic Model to Vibration Reduction in Helicopters Using an Actively Controlled Flap," Journal of the American Helicopter Society, Vol. 46, No. 1, Jan. 2001, pp. 32-43.

${ }^{23}$ Patt, D., Liu, L., and Friedmann, P. P., "Simultaneous Vibration and Noise Reduction in Rotorcraft Using Aeroelastic Simulation," Journal of the American Helicopter Society, Vol. 51, No. 2, 2006, pp. 127-140.

${ }^{24}$ Yuan, K. A. and Friedmann, P. P., Aeroelasticity and Structural Optimization of Composite Helicopter Rotor Blades with Swept Tips, NASA CR 4665, May 1995.

${ }^{25}$ Johnson, W., CAMRAD/JA - A Comprehensive Analytical Model of Rotorcraft Aerodynamics and Dynamics, Vol I. Theory Manual, Johnson Aeronautics, Palo Alto, CA, 1988.

${ }^{26}$ Johnson, W., CAMRAD/JA - A Comprehensive Analytical Model of Rotorcraft Aerodynamics and Dynamics, Vol II. Users' Manual, Johnson Aeronautics, Palo Alto, CA, 1988.

${ }^{27}$ Petot, D., "Differential Equation Modeling of Dynamic Stall," La Recherche Aérospatiale, Vol. 5, 1989, pp. 59-71.

${ }^{28}$ Shampine, L. F., Numerical Solution of Ordinary Differential Equations, Chapman and Hall, New York, 1994.

${ }^{29}$ Millott, T. A. and Friedmann, P. P., Vibration Reduction in Helicopter Rotors Using an Actively Controlled Partial Span Trailing Edge Flap Located on the Blade, NASA CR 4611, June 1994.

${ }^{30}$ Friedmann, P. P. and Shanthakumaran, P., "Optimum Design of Rotor Blades for Vibration Reduction in Forward Flight," Journal of the American Helicopter Society, Vol. 29, No. 4, 1984, pp. 70-80.

${ }^{31} \mathrm{Lim}, \mathrm{J}$. W. and Chopra, I., "Aeroelastic Optimization of a Helicopter Rotor," Journal of the American Helicopter Society, Vol. 34, No. 1, 1989, pp. 55-62.

${ }^{32}$ Jones, D. R., Schonlau, M., and Welch, W. J., "Efficient Global Optimization of Expensive Black-Box Functions," Journal of Global Optimization, Vol. 13, 1998, pp. 455-492. 
${ }^{33}$ Sóbester, A., Leary, S. J., and Keane, A. J., "On the Design of Optimization Strategies Based on Global Response Surface Approximation Models," Journal of Global Optimization, Vol. 33, 2005, pp. 31-59.

${ }^{34}$ Koch, P. N., Evans, J. P., and Powell, D., "Interdigitation for Effective Design Space Exploration using iSIGHT," Structural and Multidisciplinary Optimization, Vol. 23, No. 2, 2002, pp. 111-126.

${ }^{35}$ Johnson, W., Self-Tuning Regulators for Multicyclic Control of Helicopter Vibrations, NASA Technical Paper 1996, 1982.

${ }^{36}$ Patt, D., Liu, L., Chandrasekar, J., Bernstein, D. S., and Friedmann, P. P., "Higher-Harmonic-Control Algorithm for Helicopter Vibration Reduction Revisited," Journal of Guidance, Control, and Dynamics, Vol. 28, No. 5, September-October 2005, pp. 918-930.

${ }^{37}$ Glaz, B., Friedmann, P. P., and Liu, L., "Efficient Global Optimization of Helicopter Rotor Blades for Vibration Reduction in Forward Flight," 11th AIAA/ISSMO Multidisciplinary Analysis \& Optimization Conference, Portsmouth, VA, September 6-8 2006, pp. 1-20, AIAA Paper 2006-6997. 